\title{
Diffusion MRI Improves the Accuracy of Preoperative Diagnosis of Common Pediatric Cerebellar Tumors among Reviewers with Different Experience Levels
}

\author{
K. Koral, S. Zhang, L. Gargan, W. Moore, B. Garvey, M. Fiesta, M. Seymour, L. Yang, D. Scott, and N. Choudhury
}

\begin{abstract}
BACKGROUND AND PURPOSE: Although utility of diffusion MR imaging in the preoperative diagnosis of common pediatric cerebellar tumors is generally recognized, its added value has not been systematically studied previously. The purpose of this study was to evaluate the impact of diffusion MR imaging on the accuracy of preoperative diagnosis of common pediatric cerebellar tumors among reviewers with different experience levels.
\end{abstract}

MATERIALS AND METHODS: Review of the neuro-oncology data base yielded 96 patients whose preoperative brain MR imaging included both diffusion MR imaging $\left(b=1000 \mathrm{~s} / \mathrm{mm}^{2}\right)$ and ADC maps. There were 38 pilocytic astrocytomas, 33 medulloblastomas, 17 ependymomas, and 8 atypical teratoid/rhabdoid tumors. Six reviewers (4 residents, 2 neuroradiologists) evaluated the examinations. Two sessions were conducted with each reviewer, without and with diffusion MR imaging data on 2 separate days. The impact of diffusion MR imaging on accuracy of diagnoses was assessed.

RESULTS: In choosing the correct diagnosis of the 4 alternatives, performances of 5 of the 6 reviewers improved significantly with inclusion of the diffusion MR imaging data, from $63 \%-77 \%(P=.0003-.0233)$. The performance of 1 reviewer also improved, but the difference did not attain statistical significance $(P=.1944)$. Inclusion of diffusion MR imaging data improved the likelihood of rendering a correct diagnosis (odds ratio $=3.16,95 \%$ confidence interval $=2.07-4.00$ ) over all tumor types. When embryonal tumors were regarded as a single group, the rate of correct diagnosis increased from $66 \%-83 \%$ with diffusion MR imaging data, and performances of all of the reviewers improved significantly $(P=.0001-.05)$. The improvement in performances resulted from increased correct diagnoses of pilocytic astrocytomas, medulloblastomas, and atypical teratoid/rhabdoid tumors. There was no improvement in the correct diagnoses of ependymomas with inclusion of the diffusion MR imaging data.

CONCLUSIONS: Diffusion MR imaging improves accuracy of preoperative diagnosis of common pediatric cerebellar tumors significantly among reviewers with differing experience levels.

ABBREVIATIONS: $\quad$ AT/RT $=$ atypical teratoid/rhabdoid tumor; $\mathrm{PA}=$ pilocytic astrocytoma; WHO $=$ World Health Organization

$\mathrm{T}$ he role of diffusion MR imaging in the preoperative diagnosis of pediatric brain tumors, and in particular of cerebellar tumors, has been studied previously. ${ }^{1-4}$ The utility of diffusion MR imaging is generally attributed to the differences in the cellularity of common pediatric cerebellar tumors. Hypercellular tumors, such as medullo-

Received January 29, 2013; accepted after revision February 26

From the Departments of Radiology, University of Texas Southwestern Medical Center (K.K., W.M., B.G., M.F., M.S., L.Y., D.S.) and Children's Medical Center (K.K.) Dallas, Texas; Departments of Clinical Sciences (S.Z.) and Internal Medicine (N.C.), University of Texas Southwestern Medical Center Dallas, Texas; and Department of Neuro-Oncology (L.G.), Children's Medical Center, Dallas, Texas.

Please address correspondence to Korgun Koral, MD, Department of Radiology, University of Texas Southwestern Medical Center/Children's Medical Center, 1935 Medical District Dr, Dallas, TX 75235; e-mail: korgun.koral@utsouthwestern.edu ${ }_{2}$ Indicates Evidence-Based Medicine Level 2

http://dx.doi.org/10.3174/ajnr.A3596 blastoma and atypical teratoid/rhabdoid tumor (AT/RT), usually display restricted diffusion because of the abundant barriers generated by the cell membranes and intracellular organelles, whereas paucicellular tumors such as pilocytic astrocytomas (PAs) are characterized by large extracellular spaces resulting in fewer obstacles to water diffusion. To our knowledge, however, the additional value of diffusion MR imaging to conventional MR imaging in the accurate preoperative diagnosis of common pediatric cerebellar tumors has not been reported previously. In this study, our hypothesis was inclusion of diffusion MR imaging data in the evaluation of common pediatric cerebellar tumors improves preoperative diagnostic accuracy of reviewers, irrespective of the level of experience.

\section{MATERIALS AND METHODS}

This study was reviewed for issues of patient safety and confidentiality and was approved by the Institutional Review Board of the Uni- 
Tumor types and patient demographics

\begin{tabular}{lrlc}
\hline \multicolumn{1}{c}{ Tumor } & $\boldsymbol{n}$ & \multicolumn{1}{c}{ M:F } & Mean age, $\boldsymbol{y}$ (min-max) \\
\hline Medulloblastoma $^{2}$ & 33 & $23: 10$ & $6.49(1.15-17.19)$ \\
Ependymoma $^{\mathrm{a}}$ & 17 & $14: 3$ & $4.20(0.46-17.18)$ \\
PA & 38 & $18: 20$ & $6.86(1.44-18.73)$ \\
AT/RT & 8 & $5: 3$ & $1.01(0.25-2.29)$ \\
Total & 96 & & \\
\hline
\end{tabular}

Note:-M:F indicates male:female.

${ }^{a}$ Four anaplastic (WHO grade 3) and 13 WHO grade 2 ependymomas.

versity of Texas Southwestern Medical Center and was compliant with the Health Insurance Portability and Accountability Act.

\section{Patient Population}

Query of the neuro-oncology data base at Children's Medical Center from July 2003 through December 2011 revealed 168 patients with 1 of the 4 most common pediatric cerebellar tumors. There were 69 PAs, 67 medulloblastomas, 19 ependymomas, and 13 AT/RTs. Patients whose examinations did not include diffusion MR imaging data (both $b=1000 \mathrm{~s} / \mathrm{mm}^{2}$ images and ADC maps) were excluded. Patients whose studies had suboptimal quality diffusion MR imaging data $(n=4)$ caused by motion or artifacts generated by dental hardware were removed from the study list. The quality of the examinations was assessed by a pediatric neuroradiologist (K.K.) with 10 years of experience. The study population comprised 96 patients whose preoperative MR imaging examinations were performed at our institution and were available on PACS. All included patients underwent surgery, and tumor specimens were categorized according to the latest World Health Organization (WHO) Classification of Tumors of the Central Nervous System. The characteristics of the study population are given in Table 1 .

\section{Imaging}

The examinations were performed on 1.5T (2 Intera and 3 Achieva; $n=89$ ) and 3T (1 Achieva; $n=7$ ) scanners (Philips, Best, the Netherlands). All studies included sagittal and axial T1weighted, axial and coronal T2-weighted, and axial FLAIR (for patients $>15$ months) and gadolinium-enhanced axial, coronal, and sagittal T1-weighted sequences. Additionally, since January 2008, a gadolinium-enhanced axial FLAIR sequence has been added to the imaging protocol in patients with brain tumors for evaluation of leptomeningeal dissemination. DWI was performed with the use of a spin-echo sequence, with $b=0$ and $b=1000$ $\mathrm{s} / \mathrm{mm}^{2}$. ADC maps were generated with a monoexponential fit on a voxel-to-voxel basis for all imaging planes.

\section{Review of MR Imaging Examinations}

Four radiology residents and 2 neuroradiologists were recruited. The radiology residents (W.M., B.G., M.F., M.S.) had completed 32 months of radiology training, including 12 weeks of pediatric radiology rotation, during which they were exposed to pediatric neuroradiology. All residents had been matched to neuroradiology fellowships to commence on the completion of their residencies. The review of the examinations took place, on average 5.75 months (2-8 months), after the completion of the last part of their pediatric radiology rotations. Although it is possible that the residents may have seen a minority of the study examinations during their rotations and at conferences, they had not participated in the official read-outs for the examinations. The neuroradiologists were recruited from our institution's adult neuroradiology division; they were academic neuroradiologists practicing adult neuroradiology and were board-certified with Certificates of Added Qualification in neuroradiology. The neuroradiologists had 8 years (L.Y.) and 3 years (D.S.) of postfellowship experience. The more experienced neuroradiologist had completed her residency at our institution before the acquisition of the earliest examination in the study set. The other neuroradiologist was also a graduate of our residency program and, similar to the resident reviewers, may have seen some of the study examinations during his rotations and at conferences, but he was not one of the official readers in the radiology reports for any of the examinations. The reviewers were given relevant literature $e^{1,2,4-7}$ on the imaging of pediatric cerebellar tumors and utility of diffusion MR imaging in discriminating common pediatric cerebellar tumors at least 2 days before their first session and maintained access to this material during the review sessions.

The examinations were presented to the reviewers by a fourthyear medical student (N.C.) who did not have access to the correct diagnoses. The studies were de-identified and randomly shown to the reviewers at clinical PACS workstations (iSite PACS Version 3.6.120.0, Philips). Twelve number sets (1-96) were generated (www.randomizer.org), and a separate number set was used at each session. Two sessions (1-13 days apart) were conducted with each reviewer. The session durations were recorded. No time constraints were imposed, and the reviewers were able to spend as much time as they wished during the sessions. When breaks were taken, their lengths were recorded and subtracted from the session durations. The reviewers were able to manipulate the studies similar to a clinical readout experience. At the first sessions, preoperative brain MR imaging studies without diffusion MR imaging data and at the second sessions preoperative brain MR imaging studies including the diffusion MR imaging data were evaluated. The reviewers were asked to render a single diagnosis. The correct diagnoses and their performances were not revealed to the reviewers after the completion of the sessions. Only the age and sex of the patients were disclosed on prompting.

\section{Statistical Analysis}

The performance was evaluated on the basis of the correct diagnoses of the 4 possible choices (PA, medulloblastoma, ependymoma, and AT/RT) without and with diffusion MR imaging data. Performance was also evaluated when embryonal tumors (medulloblastoma and AT/RT) were grouped together without and with diffusion MR imaging data. We used mixed logistic models to evaluate the effect of diffusion MR imaging data. The indicators of diffusion MR imaging data and resident/ neuroradiologist were included as fixed effects. These models also included random effects to account for the multilevel correlation among observations obtained from the same patient or the same reviewer. A value of $P<.05$ was taken to represent statistical significance. All statistical analyses were performed with the use of SAS 9.3 (SAS Institute, Cary, North Carolina).

\section{RESULTS}

In choosing the correct diagnosis of the 4 alternatives, the performances of 5 of 6 reviewers improved significantly with the addi- 

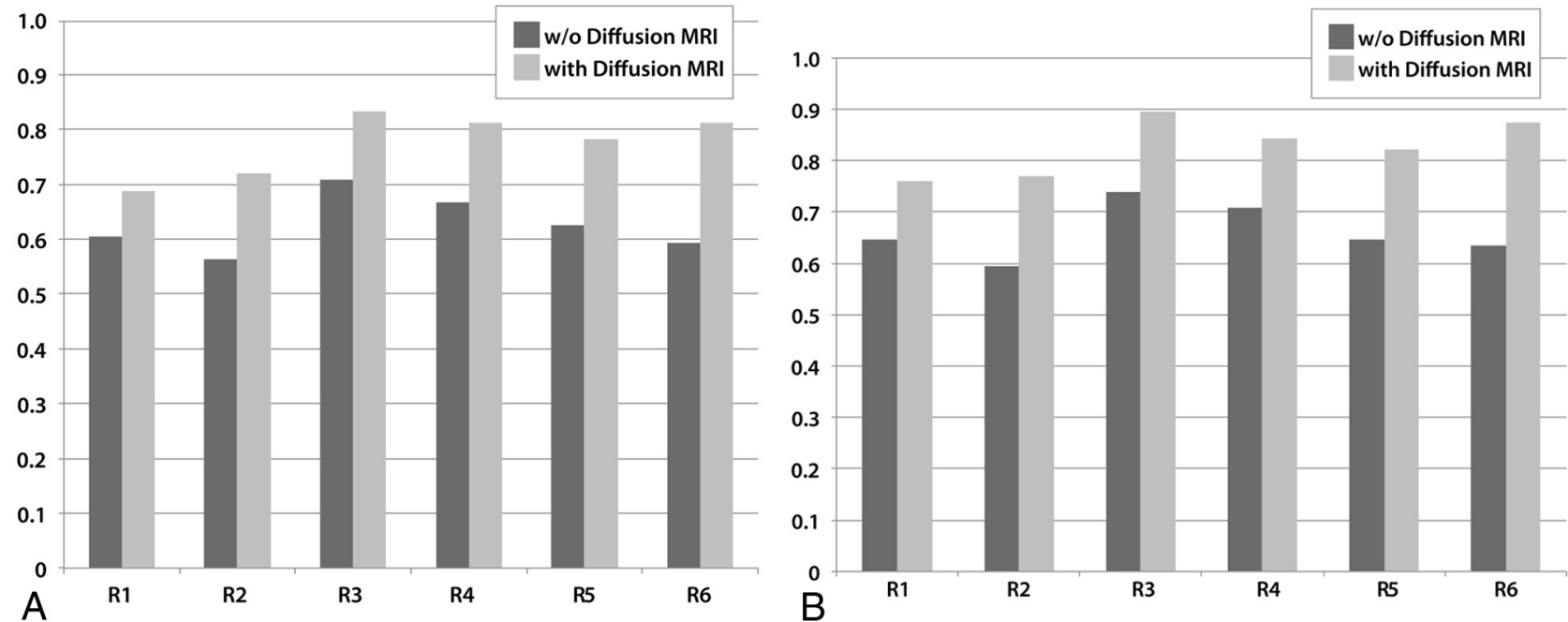

FIG 1. Graphs demonstrate the performances of the reviewers (R1-R4: residents, R5-R6: neuroradiologists) without (dark gray) and with (light gray) diffusion MR imaging data. A, When 4 choices are considered: PA, medulloblastoma, ependymoma, and AT/RT. B, When 3 choices are considered: embryonal tumors (medulloblastoma and AT/RT), ependymoma, and PA.

\begin{tabular}{|c|c|c|c|c|c|}
\hline \multirow{2}{*}{ with Diffusion MRI } & \multicolumn{4}{|c|}{ Diagnosis } & \multirow{2}{*}{ Total } \\
\hline & AT/RT & Ependymoma & Medulloblastoma & PA & \\
\hline AT/RT & & 8 & 11 & & \\
\hline Ependymoma & & 81 & 6 & & \\
\hline Medulloblastoma & & & 98 & & \\
\hline PA & & 3 & 31 & & \\
\hline Total & & & 1 & & \\
\hline
\end{tabular}

A

\begin{tabular}{|c|c|c|c|c|}
\hline \multirow{2}{*}{$\begin{array}{l}\text { w/o Diffusion MRI } \\
\text { with Diffusion MRI }\end{array}$} & \multicolumn{3}{|c|}{ Diagnosis } & \multirow{2}{*}{ Total } \\
\hline & Ependymoma & Embryonal & $\mathrm{PA}$ & \\
\hline Ependymoma & 81 & 16 & & \\
\hline Embryonal & 84 & & & \\
\hline PA & 31 & 37 & & \\
\hline Total & & & & \\
\hline
\end{tabular}

B

FIG 2. Impact of inclusion of diffusion MR imaging data to correct diagnoses. A, For 4 tumor categories. A total of 1,152 diagnoses ( 96 tumors $\times 6$ reviewers $\times 2$ sessions) were rendered. $B$, For embryonal tumors (medulloblastomas and AT/RTs combined), ependymomas, and PAs.

tion of the diffusion MR imaging data $(P=.0003-.0233)$. The performance of the remaining reviewer (a resident) also improved, but the difference did not attain statistical significance
$(P=.1944)$. The overall rate of correct diagnosis increased from $63 \%-77 \%$ with the addition of DWI data (Fig $1 A$ ).

When embryonal tumors (medulloblastoma and AT/RT) were regarded as a single group, the overall rate of correct diagnosis increased from 66\%-83\% with the inclusion of the diffusion MR imaging data. In this case, all of the reviewers showed statistically significant improvement with the addition of DWI data $(P=.0001-.05)$ (Fig $1 B)$.

When 4 choices (PA, medulloblastoma, ependymoma, AT/RT) were considered, inclusion of the diffusion MR imaging data significantly improved the likelihood of rendering a correct diagnosis (odds ratio $=3.16,95 \%$ confidence interval $=2.07-4.00)$ over all tumor types (Figs 2 and 3). By including the interaction between the diffusion MR imaging data and tumor categories into the model, we showed that there was no significant difference in the improvement effect of diffusion MR imaging data on the correct diagnosis of PAs, medulloblastomas, and AT/RTs. Of the 102 (17 ependymomas $\times 6$ reviewers) possible correct ependymoma diagnoses, 81 correct diagnoses were provided both without and with diffusion data, indicating correct ependymoma diagnoses did not improve with the inclusion of the MR imaging diffusion data.

The mixed model suggested that there was no significant difference between the overall performances and performances with- 

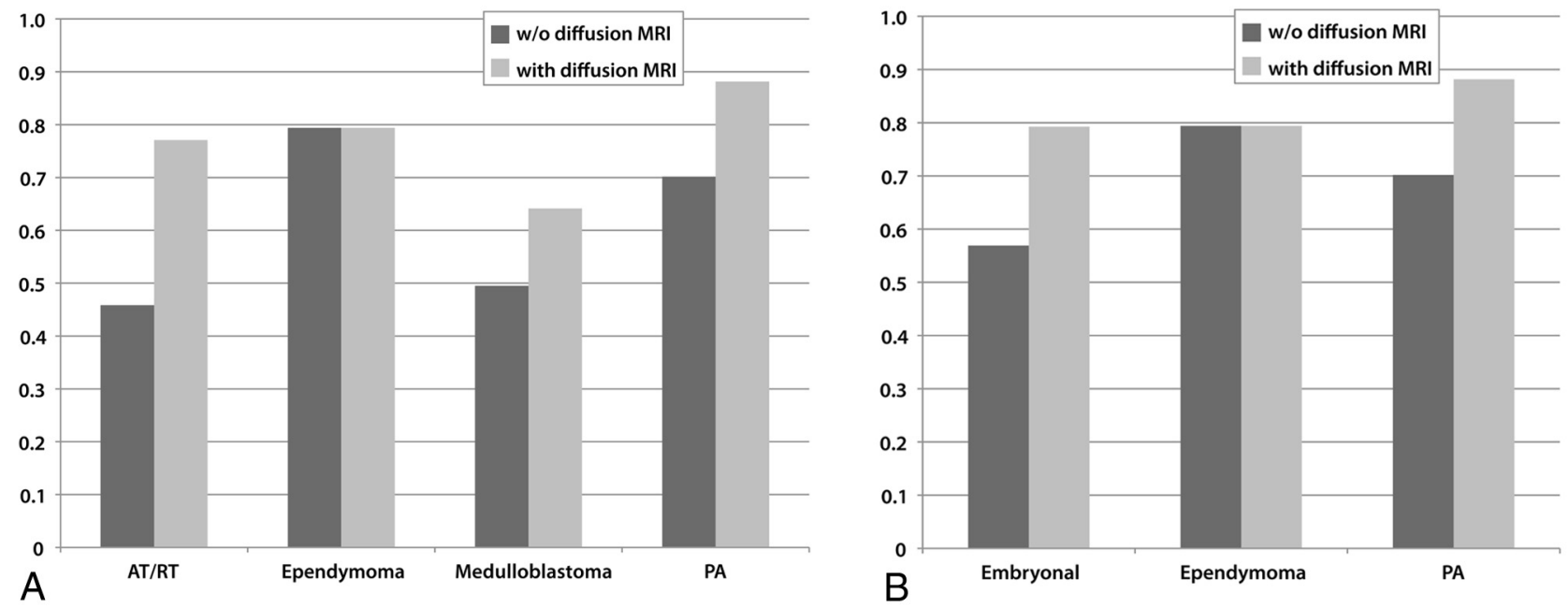

FIG 3. Graphs depict the percentages of correct diagnoses for tumor categories without (dark gray) and with (light gray) diffusion MR imaging data. A, When 4 choices are considered: PA, medulloblastoma, ependymoma, and AT/RT. B, When 3 choices are considered: Embryonal tumors (medulloblastoma and AT/RT), ependymoma, and PA.
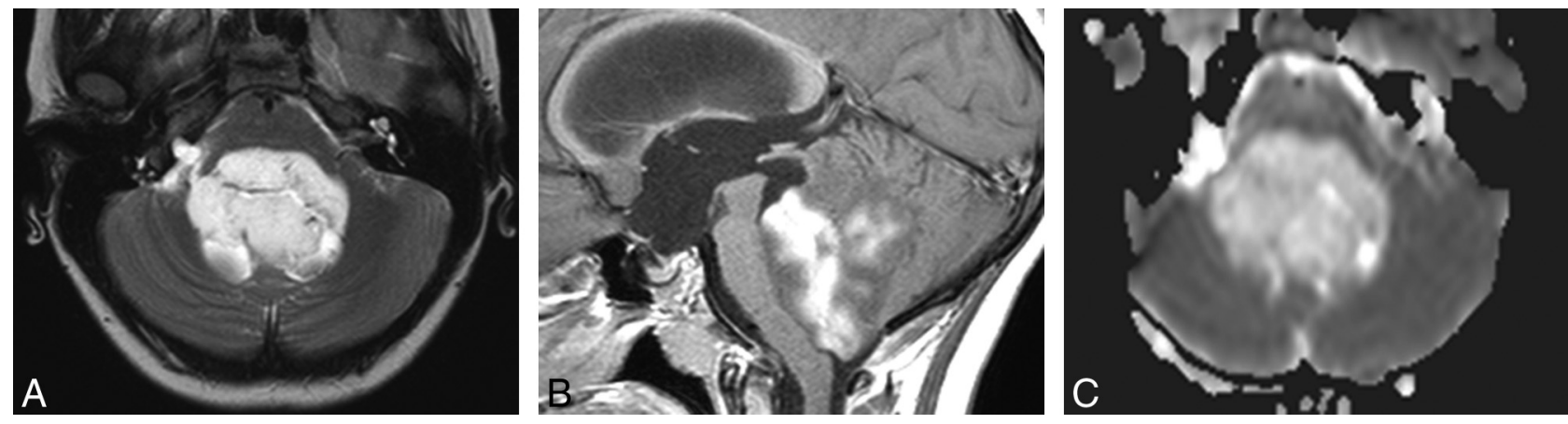

FIG 4. PA in a 10-year-old girl. Without diffusion MR imaging data, 5 reviewers were incorrect. Four selected ependymoma and 1 selected medulloblastoma. With diffusion MR imaging data, 5 reviewers were correct. One reviewer (a neuroradiologist) still chose ependymoma. A, Axial T2-weighted image displays a markedly hyperintense tumor. B, Sagittal gadolinium-enhanced T1-weighted image demonstrates intense and heterogeneous enhancement of the mass. C, ADC map shows facilitated diffusion within the mass compared with the uninvolved cerebellum.

out and with diffusion MR imaging data among the radiology residents and the neuroradiologists. The session durations were $121.2 \pm$ 31.9 minutes (min-max, 81-170) and $120.8 \pm 40.7$ minutes (minmax, 80-170) for those without diffusion MR imaging data and with diffusion MR imaging data, respectively.

\section{DISCUSSION}

Tumors of the posterior fossa comprise approximately $50 \%$ of intracranial tumors in children. ${ }^{8}$ When brain stem tumors and choroid plexus papillomas are excluded, PAs, medulloblastomas, ependymomas, and AT/RTs make up approximately $93 \%$ of pediatric cerebellar tumors. ${ }^{1}$ Therefore, when a new pediatric cerebellar tumor is encountered, it is very likely that the diagnosis will be 1 of these 4 tumors. Conventional MR imaging findings of common cerebellar tumors are well known. ${ }^{5,9-11}$ PAs are characteristically well delineated, with markedly T2-weighted hyperintense solid components that may show intense enhancement with intravenous gadolinium administration. Cysts with enhancing mural nodules are associated with PAs. Ependymomas are known for their spread on the surfaces of the cerebellum and brain stem. Medulloblastomas and AT/RTs have similar conventional MR imaging findings: Both tumor categories are relatively hypoin- tense on T2-weighted images. Medulloblastomas are more commonly midline tumors, and AT/RTs are seen in younger children (Figs 4-6). The performance of our reviewers is similar to what was reported in a study of 33 pediatric cerebellar tumors. ${ }^{12}$ In this study, a pediatric neuroradiologist chose the correct diagnosis, without diffusion MR imaging data, $73 \%$ of the time among 3 groups: primitive neuroectodermal tumors, astrocytomas, and ependymomas/others. ${ }^{1} \mathrm{H}$-MR spectroscopy has also been used in the preoperative diagnosis of pediatric cerebellar tumors. In a study of 20 children with cerebellar tumors, reviewers blinded to the histopathologic diagnoses achieved high diagnostic accuracy-approximately $90 \%$ - with the use of ${ }^{1} \mathrm{H}-\mathrm{MR}$ spectroscopy data. ${ }^{13}$

Diffusion MR imaging offers an important utility in the preoperative diagnosis of pediatric cerebellar tumors, primarily because of the vastly different cellularity and extracellular architecture of the common tumor types. For example, PAs are relatively paucicellular neoplasms with large extracellular spaces. ${ }^{14}$ These features are likely to be responsible for the facilitated diffusion that PAs display. ${ }^{1,2,4,15}$ Conversely, medulloblastomas and AT/RTs are hypercellular tumors with diminutive extracellular matrices, resulting in relatively decreased ADC val- 

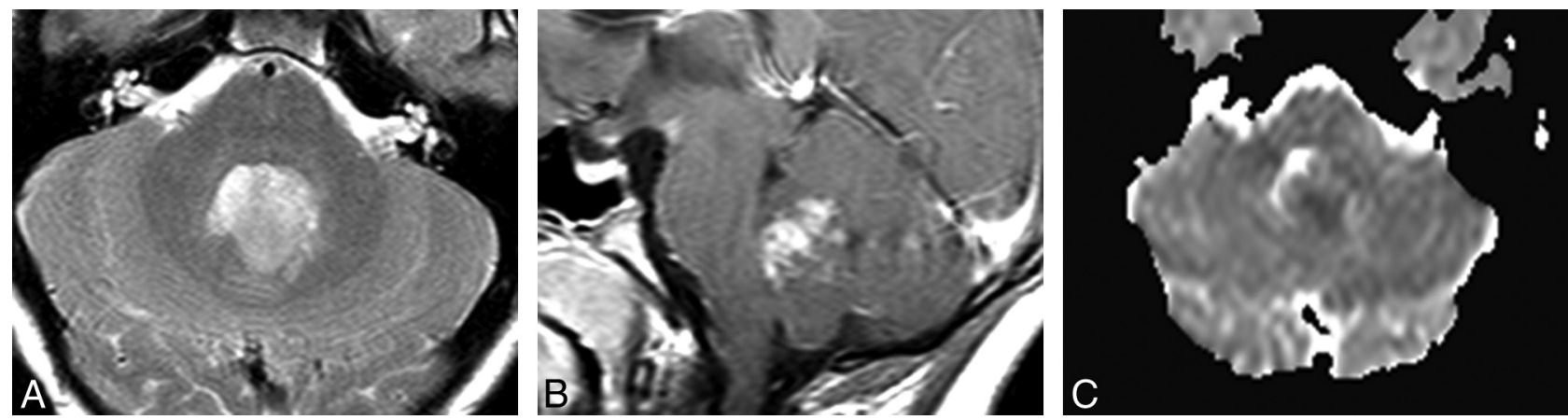

FIG 5. Medulloblastoma in a 12-year-old boy. Without diffusion MR imaging data, 4 reviewers ( 3 residents and 1 neuroradiologist who chose ependymoma) were incorrect. With diffusion MR imaging data, all reviewers were correct. $A$, Axial T2-weighted image shows that the tumor in the fourth ventricle is relatively hypointense. B, Sagittal gadolinium-enhanced T1-weighted image shows that the tumor enhances intensely and heterogenously. C, ADC map shows that the tumor is slightly hypointense to isoinstense compared with the normal cerebellum.
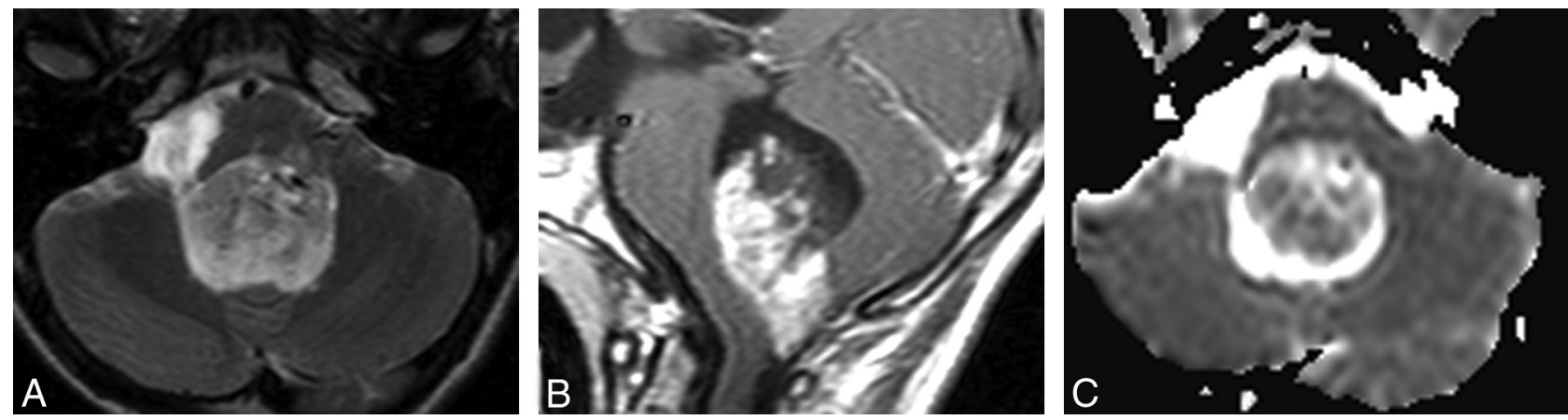

FIG 6. Ependymoma (WHO grade 2) in a 15-year-old boy. Without diffusion MR imaging, all reviewers were correct. With diffusion data, 3 reviewers (2 residents and 1 neuroradiologist) changed their diagnoses to medulloblastoma. $A$, Axial T2-weighted image demonstrates a hyperintense mass extending toward the right foramen of Luschka. B, Sagittal gadolinium-enhanced Tl-weighted image shows that the tumor is heterogeneous, but intense with enhancement. C, ADC map shows that the mass is isointense to slightly hyperintense compared with normal cerebellum.

ues and hyperintensity on $b=1000 \mathrm{~s} / \mathrm{mm}^{2}$ or exponential diffusion images. ${ }^{15-17}$

Although the usefulness and limitations of diffusion MR imaging in the preoperative diagnosis of cerebellar tumors have been studied previously, ${ }^{1,2,4,17,18}$ to our knowledge, there are no publications that evaluated the additional value of diffusion MR imaging in improving the accuracy of preoperative diagnosis in this context. Our results show that inclusion of diffusion MR imaging into the imaging protocol significantly improved the performance of all but 1 reviewer (a resident) in choosing the correct diagnosis of the 4 possible choices. This reviewer's performance improved as well, but the improvement did not reach statistical significance. We found that the percentage of correct ependymoma diagnosis did not change with the inclusion of the diffusion MR imaging data. The improvement in the performances of the reviewers with usage of the diffusion MR imaging data resulted from increased correct diagnoses of PAs, medulloblastomas, and AT/RTs. Difficulty in deciding whether ependymomas displayed restricted, facilitated, or normal (ie, isointense to normal brain on ADC maps) diffusion may have contributed to the lack of improvement in the evaluation of ependymomas with the diffusion MR imaging data.

It is justifiable to consider the embryonal tumors (medulloblastoma and AT/RT) of the posterior fossa as a single group, given their very similar imaging characteristics on conventional MR imaging and diffusion MR imaging. The main discriminators between medulloblastomas and cerebellar AT/RTs are the age of the patient at presentation and, to a lesser extent, the location of the tumor. When choices were between embryonal tumors, ependymoma, and PA, there was statistically significant improvement in the performances of all the reviewers with inclusion of the diffusion MR imaging data. The improvement in performance was achieved without additional cost in time to arrive at a diagnosis.

The lack of statistically significant difference between the performances and improvements in the performances of the residents and neuroradiologists may be attributed to the relative ease with which to distinguish the characteristics of the common pediatric cerebellar tumors. The more recent exposure of the residents to pediatric neuroradiology at a tertiary referral center in which there is a busy neuro-oncology program may have contributed to their performances favorably. At our pediatric neuroradiology service, during read-outs with residents and at teaching sessions, the utility of diffusion MR imaging in the diagnosis of pediatric cerebellar tumors is discussed emphatically. The neuroradiologists, on the other hand, were exclusively practicing adult neuroradiology without routine exposure to pediatric neuroradiology.

One potential limitation of our study is the exclusion of posterior fossa tumors other than the 4 most common cerebellar tumors. The justification for the exclusion of brain stem tumors is the relative ease with which to distinguish them from the cerebel- 
lar tumors; therefore brain stem tumors are not generally considered in the differential diagnosis of pediatric cerebellar tumors. The reason for the exclusion of the choroid plexus tumors was 2-fold: First, their imaging characteristics_-intensely enhancing intraventricular masses with nodular surface features resembling a frond-allow for relatively easy discrimination of choroid plexus tumors from other tumors of the posterior fossa. Second, they are relatively infrequent compared with the 4 tumor categories included in this series. Gangliogliomas were also excluded as they rarely involve the cerebellum in children. ${ }^{1}$ In a report of 10 gangliogliomas (including 2 patients under the age of 18 years), the minimum ADC values were provided. ${ }^{19}$ These values appear to approximate values reported for PAs. ${ }^{1,2,4}$ Although gangliogliomas of the cerebellum may be considered in the differential diagnosis of a pediatric cerebellar mass that shows facilitated diffusion, given their low incidence, in practice, they are rarely included.

Whether seeing the examinations for the second time improved the performance independent of the diffusion MR imaging data may be considered as a confounding factor and thus a limitation; however, because the correct diagnoses were not disclosed after either the first or the second session, the impact of this probably is minimal, if any. Additionally, recall is not considered a factor in the study design because the correct diagnoses and information on the reviewers' performances were not given after the first session.

It is also important to emphasize that the purpose of our study was not to demonstrate the utility of diffusion MR imaging in the preoperative diagnosis of common cerebellar tumors but to show the improvement, if any, provided by the inclusion of the diffusion MR imaging data into the imaging protocol among reviewers with different experience levels. Therefore, we believe that it was reasonable to limit the possible diagnoses offered to the reviewers to the most common pediatric cerebellar neoplasms for which usefulness of diffusion MR imaging was previously described.

\section{CONCLUSIONS}

Our results show that inclusion of diffusion MR imaging data in the imaging protocol significantly improves the accuracy of preoperative diagnosis of common pediatric cerebellar tumors among reviewers with different experience levels. The improvement results from increased correct diagnoses of PAs, medulloblastomas, and AT/RTs. We found no impact of diffusion MR imaging on the correct diagnosis of ependymomas. The improvement in the performance of the reviewers was achieved with minimal additional penalty in imaging time ( $<1$ minute) and without penalty in interpretation duration.

\section{REFERENCES}

1. Gimi B, Cederberg K, Derinkuyu B, et al. Utility of apparent diffusion coefficient ratios in distinguishing common pediatric cerebellar tumors. Acad Radiol 2012;19:794-800

2. Jaremko JL, Jans LB, Coleman LT, et al. Value and limitations of diffusion-weighted imaging in grading and diagnosis of pediatric posterior fossa tumors. AJNR Am J Neuroradiol 2010;31:1613-16

3. Kan P, Liu JK, Hedlund G, et al. The role of diffusion-weighted magnetic resonance imaging in pediatric brain tumors. Childs Nerv Syst 2006;22:1435-39

4. Rumboldt Z, Camacho DL, Lake D, et al. Apparent diffusion coefficients for differentiation of cerebellar tumors in children. AJNR Am J Neuroradiol 2006;27:1362-69

5. Koral K, Gargan L, Bowers DC, et al. Imaging characteristics of atypical teratoid-rhabdoid tumor in children compared with medulloblastoma. AJR Am J Roentgenol 2008;190:809-14

6. Poretti A, Meoded A, Huisman TA. Neuroimaging of pediatric posterior fossa tumors including review of the literature. J Magn Reson Imaging 2012;35:32-47

7. Raybaud C, Barkovich AJ. Intracranial, orbital, and neck masses of childhood. In: Barkovich AJ, Raybaud C, editors. Pediatric Neuroimaging. 5th ed. Philadelphia: Lippincott Williams \& Wilkins; 2012:637-807

8. Harwood-Nash DC. Primary neoplasms of the central nervous system in children. Cancer 1991;67:1223-28

9. Koeller KK, Rushing EJ. From the archives of the AFIP: pilocytic astrocytoma: radiologic-pathologic correlation. Radiographics 2004;24:1693-708

10. Smith AB, Smirniotopoulos JG, Horkanyne-Szakaly I. From the radiologic pathology archives: intraventricular neoplasms: radiologic-pathologic correlation. Radiographics 2013;33:21-43

11. Yuh EL, Barkovich AJ, Gupta N. Imaging of ependymomas: MRI and CT. Childs Nerv Syst 2009;25:1203-13

12. Arle JE, Morriss C, Wang ZJ, et al. Prediction of posterior fossa tumor type in children by means of magnetic resonance image properties, spectroscopy, and neural networks. I Neurosurg 1997;86:755-61

13. Harris LM, Davies N, Macpherson L, et al. The use of short-echotime ${ }^{1} \mathrm{H}$ MRS for childhood cerebellar tumours prior to histopathological diagnosis. Pediatr Radiol 2007;37:1101-09

14. Scheithauer BW, Hawkins C, Tihan T, et al. Pilocytic astrocytoma. In: Louis DN, Ohgaki H, Wiestler OD, et al, eds. WHO Classification of Tumours of the Central Nervous System. Lyon, France: International Agency for Research on Cancer: 2007:14-21

15. Gauvain KM, McKinstry RC, Mukherjee P, et al. Evaluating pediatric brain tumor cellularity with diffusion-tensor imaging. $A J R A m J$ Roentgenol 2001;177:449-54

16. Yamashita $Y$, Kumabe $T$, Higano $S$, et al. Minimum apparent diffusion coefficient is significantly correlated with cellularity in medulloblastomas. Neurol Res 2009;31:940-46

17. Rumboldt $Z$. The Holy Grail and the quest for the gold standard. AJNR Am J Neuroradiol 2010;31:1617-18

18. Forbes JA, Reig AS, Smith JG, et al. Findings on preoperative brain MRI predict histopathology in children with cerebellar neoplasms. Pediatr Neurosurg 2011;47:51-59

19. Kikuchi T, Kumabe T, Higano S, et al. Minimum apparent diffusion coefficient for the differential diagnosis of ganglioglioma. Neurol Res 2009;31:1102-07 